\title{
Decision spread in the corporate board network
}

\author{
Stefano Battiston ${ }^{(1)}$ Gérard Weisbuch ${ }^{(1)}$ and Eric Bonabeau (2) \\ (1) Laboratoire de Physique Statistique, ENS, 24 rue Lhomond, 75005 Paris, France \\ (2) Icosystem Corp., 545 Concord Av. Cambridge, MA 02138, USA \\ Correspondence to: stefano.battiston@ens.fr, tel +33144323623 , fax +33144323433
}

February 2, 2008

\begin{abstract}
Boards of large corporations sharing some of their directors are connected in complex networks. Boards are responsible for corporations' long-term strategy and are often involved in decisions about a common topic related to the belief in economical growth or recession.

We are interested in understanding under which conditions a large majority of boards making a same decision can emerge in the network. We present a model where board directors are engaged in a decision making dynamics based on "herd behavior". Boards influence each other through shared directors.

We find that imitation of colleagues and opinion bias due to the interlock do not trigger an avalanche of identical decisions over the board network, whereas the information about interlocked boards' decisions does. There is no need to invoke global public information, nor external driving forces.

This model provides a simple endogenous mechanism to explain the fact that boards of the largest corporations of a country can, in the span of a few months, take the same decisions about general topics.
\end{abstract}

PACS: 89.75 -k; 89.65 -s

Keywords: social networks, opinion dynamics, directorate interlock, Ising model.

\section{Introduction}

We present a model for the diffusion of decisions in a network of corporate boards linked by shared board directors. Boards of large corporations share common directors with each other forming complex networks, often characterized by Small World properties [3].

An example of board network is shown in figure 1: nodes represent boards of directors, two boards are connected by an edge if there is at least one director sitting on both boards at the same time. The network represents the boards 
at 1 degree of separation (in term of edges) away from the board of Chase Manhattan Bank. For instance the boards of Abbott Laboratories and Chase Manhattan Bank are connected because they share one director.

We are interested in the influence of such networks on the decisions made by boards. The role of boards is to make decisions about the long-term strategy of the corporations. There are essentially two kinds of decisions a board is faced to:

- Decisions regarding topics specific to the board, such as the appointment of a vice president, for which we can assume that different boards don't influence each other. We previously studied [1] the role of subsets of well connected directors on decisions of this type.

- By contrast, there are decisions about topics of general interest to the economy such as whether to increase or decrease investments in development or in advertisement, which depend on the belief in economical growth or recession. Decisions of equivalent generality concern the adoption of governance practices. In these cases, decisions previously made in some boards might influence other boards, through the presence of shared directors. The present paper is devoted to the dynamics of this kind of decisions across the board network.

The second issue has already been studied in sociology. Haunschild in an article [9] about the impact of interlock on US corporate acquisition activity in the 80', demonstrates the role of inter-organizational imitation of managers. The article by Davis and Greve [4] concerns the diffusion of corporate governance practices such as the "poison pill" among the US largest corporations in the 80' (The poison pill is a counter measure against hostile takover allowing "target shareholders to acquire shares at a 50 perc. discount if an acquirer passes a certain ownership threshold").

Davis and Greve analyse a large set of empirical data and show that the poison pill was primarily mediated by director interlock, with a spreading time scale of the order of one year. In order to explain the observed spreading dynamics, the authors develop a contagion model in which boards are agents with a certain probability to imitate an earlier adopter of the practice. The influence of a board on an interlocked board is reduced or enhanced by a number of factors, discussed in detail by the authors, such as similar industrial sector, firm size, firm performance, concentration of ownership of both companies involved.

We here present a model where agents are instead board directors engaged in a decision making dynamics. Inter-boards influence takes place through shared directors. We are not taking into account the heterogeneity of inter-boards influences. We are interested in understanding under which conditions a large majority of boards making a same decision can emerge. The decision making process is based on the hypothesis that agents tend to follow the majority of the agents to whom they are connected.

This kind of behavior is not perfectly rational in the sense of economics and is known as "herd behavior" [10] [6], but presents formal analogies with the Ising model often used for social system models, [7] [8] [11].

Models [7] [8] [11] are based on binary opinion dynamics. The basic updating process is the same as described here by equations 1 and 2, but interaction 
topologies are simplified to either full connectivity or lattice connectivity.

Unlike the cited works, in the present model the dynamics takes place on an empirical etherogeneous network organized in interconnected groups. This is the director network of Fortune 1000 corporations (data are kindly provided by Davis [3]). The connectivity within a group (a board of directors) and between groups is highly etherogeneous. Moreover, the dynamics takes places in different groups at different times.

Furthermore, we explore two different scenarios for the influence exerted by groups upon each other. In the first scenario the information about other groups' decisions only affects the initial opinions of interlocking directors. In the second scenario such information is taken into account by all directors during the whole board meeting.

Our main result in this paper is that the way this information is taken into account by directors at a local level, determines whether, at a global level, it can emerge a large majority of boards making the same decision.

\subsection{Building a model for the spread of decisions}

We here focus on a time scale of a few months, just the time for boards to hold a couple of meetings. Typically we have in mind fast decisions concerning investment and advertisement strategies for the next six months. Decisions of this type are relevant for the macro-economics of a country, especially if corporations tend to make similar decisions in connection to external dramatic events such as crises or wars. It is therefore important to understand what structural parameters of the network determine the number of boards adopting a given decision. Of course this is a formidable task to accomplish, so we start considering in this paper a simple situation in which decisions are binary and the most central board of the network takes a decision in the first place and the other boards meet afterwards. Because of the interlock, the decision made in a given board is influenced by the presence of some directors that were present at a previous meeting on another board.

Let us assume that each board has to make a binary decision. The opinion of director $i$ is represented by a binary variable $s_{i}= \pm 1$. For the decision making dynamics in each board meeting, we assume a survey-like mechanism, as in [1]: each director updates his opinion based on the average opinion of the other directors, according to a logit probability function (see below).

Boards have meetings once per month, according to a given schedule. Directors start from an initial opinion $s_{i}= \pm 1$ at the beginning of the meeting. By the end of a meeting, directors adopt the opinion that agrees with the decision made by the board.

Therefore, directors serving on several boards start a new meeting with an initial opinion adopted at the last meeting in another board. In this way the decision made previously in a board can influence the decision making dynamics in another board.

After first studying the decision spread on some simple test-bed networks we concentrated on the real network of the boards 1 and 2 degrees of separation away from the board of Chase Manhattan Bank in 1999. 
The most central board of the network meets first and the other boards meet afterwards, according to a given schedule.

The topological structure of the network is known from the names of the directors in the boards of the set of companies under consideration. On the other hand, we do not know the magnitude of the influence of a board on another one.

We here assume that the influence of one board on a connected board varies in accordance with the number of directors shared by the two boards (the interlock). For sake of simplicity we don't take into account those specific factors such as: some boards are very influential over other ones because of differences in prestige, revenue, economic performance, expertise in a specific field, see [4] for a detailed analysis.

We here present a first model in which the influence of a board $b_{1}$ on another board $b_{2}$ is due only to the fact that those directors of $b_{1}$ which sit also in $b_{2}$, will support, at the beginning of the meeting in $b_{2}$, the choice adopted by $b_{1}$. We study how the pattern of decisions depends on the value of the average interlock.

In the second model the influence of the interlock is increased by two factors.

- Inside the board, directors sitting also on common outside boards have a larger mutual influence than other directors.

- Decisions made by other connected boards can be taken into account as external signals by all board members.

\section{$2 \quad$ Model 1}

\subsection{Inside board dynamics}

We here describe the simplest model. Directors meet, discuss and vote.

In any new meeting directors' opinions are initialized as follows:

- Directors participating at a meeting for the first time are initialized with opinions randomly chosen among 0 or 1 ;

- all other directors start with the opinion they acquired at the previous meeting they participated at,

- except for directors from the most central node $b_{c}$ which start at any new meeting with the opinion that won in $b_{c}$.

At each time step, a director randomly selected is informed of the opinions of all other directors in the board at time t, and average them to evaluate a field $h_{i}$ :

$$
h_{i}=\frac{1}{n} \sum_{j=1}^{n} s_{j}
$$

$n$ being the size of the board. He updates his opinion according to a probabilistic choice function of the field. The probability that director $i$ takes some opinion \pm 1 at time $t+1$ is given by:

$$
P\left\{s_{i}(t+1)= \pm 1\right\}=\frac{\exp \left( \pm \beta h_{i}(t)\right)}{\exp \left(\beta h_{i}(t)\right)+\exp \left(-\beta h_{i}(t)\right)}
$$


The updated opinion is expressed by the director and is taken into account by later sampled directors.

The average opinion $m$ of the board, is a function of time during each meeting and eventually converges at a value $m^{*}= \pm 1$ for $\beta \gg 1$. In all simulations we set $\beta=4$ such that the board converges to unanimity in less than 30 steps, i.e. in roughly three average sampling of each director. We take the final value of $m^{*}$ as the decision of the board. We will write $m$ for $m^{*}$ in the following for sake of simplicity.

\subsection{Boards' network dynamics}

The central node meets first and the decision made is by convention +1 .

Boards are assumed to meet monthly and to discuss again the same topic. Each step represents a month, and hence a cycle of board meetings. The central board meet first, followed by the nodes on each surrounding layer, in order of distance from the center. Unless specified, the meeting schedule described here will be assumed in the following for model 1 and for model 2 .

Within the model described so far, the whole network converges to a stable value of the average decision $M^{*}$ in $2-3$ steps, which is reasonable. In fact it is conceivable that some actions require more than one decisional step, to be fully approved.

If a board happens to make a decision -1, it is quite reluctant to change in the next. This model differs from usual contagion models in that the risk of infection or adoption of an innovation may be diminished by an internal dynamics of the board.

We will write $M$ for $M^{*}$ in the following for sake of simplicity. We run several simulations with varying random seeds per set of parameters and we monitor the following variables:

- The average decision $\left\langle m_{k}\right\rangle$ of the board $k$. Because $m_{k}= \pm 1$ at each run, the probability $p_{k}^{+}$that $m_{k}=+1$ is given by: $p_{k}^{+}=\frac{1+<m_{k}>}{2}$

- The average decision $M$ of the whole board network over several runs. We will call $P^{+}$the fraction of boards that make decision +1 , averaged over several runs.

\section{$3 \quad$ Results}

We present the results of model 1 on different networks of increasing connectivity. First, with consider a square lattice (figure 2) with an odd number of vertices on the edge, so that there is a node in the center of the lattice. A node represents a board of $n=10$ directors, a link represents the existence of some shared directors between two boards. The fraction of shared directors between two connected boards is a parameter $\gamma$ that we can control. For each pair of connected boards we can impose that they share a larger fraction of directors by replacing a director of the first board with a director of the second one (provided that each director sits only on one chair!). It is very important to remark that increasing the number of shared directors per pair of boards, introduces 
at some point some links between boards that were not connected before. For instance at the end when each board is sharing all its directors with its four initial neighbouring boards, in fact all boards must share the same 10 directors and therefore the graph must be complete. With no interlock, all nodes are isolated and $\gamma=0$.

The fraction $P^{+}$of boards that adopted decision +1 is plotted as a function of $\gamma$ in figure 4 (circles).

The same procedure has been applied to a star network (figure 3) and to the network of boards 1 degree of separation away from the CMB's board (figure 1). Plots of $P^{+}$are shown in figure 4(triangles and squares, respectively).

$P^{+}$increases linearly with the average fraction $\gamma$ of directors shared by any two boards until it saturates for $\gamma$ around 0.6. The value of $\gamma$ observed in real board networks is $\gamma=.1 \pm .02$, which corresponds to $P^{+} \simeq 0.6$, i.e. 60 per cent chances to adopt decision +1 , compared to 50 per cent chances in absence of interlock $\left(P^{+}=0.5\right.$ for $\left.\gamma=0\right)$.

As a first result, within the hypothesis of this model the degree of interlock observed in the real CMB's network is low compared to the one required to a have a large majority of boards adopting a same decision. Results will be further analysed in the discussion section.

\section{Model 2: the influence of lobbies and in- formation}

The second model shares the same general dynamics as the first model in terms of initial conditions and sequences of updating directors and boards opinion, but the field variables $h_{i}$ are increased by two terms taking into account mutual influence of well-connected directors and the propagation of information from boards which met previously.

The central question about the role of the interlock is how it influences the decision making dynamics ([2], [5]). In a previous paper ([1]) we proposed a mechanism that we re-introduce in the present model. We assume that two directors in the board who also serve in another outside board are likely to take each other's opinion into account more seriously than the opinion of directors with whom they don't have additional professional relationships. These directors form a graph called interlock graph of the board, for simplicity called "lobby" in the following. In the equation for the field felt by directors who participate in a lobby we introduce a coupling factor. Director $i$ feels a field $h_{i}^{0}$ defined as follows:

$$
h_{i}^{0}=\frac{1}{n} \sum_{j=1}^{n}\left(1+\alpha_{1} J_{i j}\right) s_{j}
$$

where $\mathrm{n}$ is the size of the board, $J_{i, j}$ is the number of outside boards on which directors $i$ and $j$ sit together, $\alpha_{1}$ is a parameter modulating the mutual influence of directors in the lobby. 
Of course directors take into account their own opinion: by definition $J(i, i)$ should be the total number of outside boards where director $i$ sits. This is much larger than the number of outside boards a director can share with another one. So in order to avoid giving a too big weight to the opinion of a director himself compared to the opinion of his colleagues, $J(i, i)$ is set as the number of outside boards where director $i$ serves with at least one other director of the board. In summary, a director with several appointments, takes into account his own opinion more than that of his colleagues. Among his colleagues then, he takes into account their opinions based on how many professional relationships he holds with them.

As a consequence of the dynamics, directors belonging to a connected component of a lobby tend to have the same opinion. As the lobby is a graph consisting of one or more connected components, it must be kept in mind that different connected components may have different opinions and their effects on the whole board could partly cancel out.

Furthermore, information about what decision other boards have made so far, can reach a board thanks to the interlocking directors. Therefore, their influence is twofold: on one hand they have an initial opinion which reflects the decision made in another board (model 1). They could just support that same decision, keeping confidential the information about the fact that the other board has made that decision. On the other hand they can reveal this information to the other directors, which will take it into account to form their opinion. We model this by means of a second term in the equation for the field. This is a sort of external field $h^{e}$ acting on all directors of board $k$, but specific to the board $k$. It equals the sum of the decisions made so far in the boards connected through interlock to the board $k$, weighted by the number of shared directors:

$$
h^{e}=\sum_{l=1}^{\operatorname{deg}(k)} J_{k l}^{B} b_{l}
$$

The sum is running on the boards interlocked to board $k, \operatorname{deg}(\mathrm{k})$ is the connectivity degree of board $k, J_{k l}^{B}$ is the number of directors shared by boards $k$ and $l, b_{l}$ is the decision made by the board if it has already met, $b_{l}=0$ otherwise. Again we don't include any factor of prestige for the boards. Nevertheless, a board is more influential on another if it has more directors on it. It is important to stress the fact that boards that have not yet met do not influence the decision.

The information reaching a board can then be different from the information about what all other boards, interlocked or not, have decided so far.

The total field acting on director $i$ is:

$$
h_{i}=h_{i}^{0}+h^{e}=\frac{1}{n} \sum_{j=1}^{n}\left(1+\alpha_{1} J_{i j}\right) s_{j}+\alpha_{2} \sum_{l=1}^{\operatorname{deg}(k)} J_{k l}^{B} b_{l}
$$

where $\alpha_{2}$ is a parameter modulating the influence of the information about 
other board's decisions.

We are now interested in the behavior of the system in the space of the parameters $\alpha_{1}$ and $\alpha_{2}$.

\section{$5 \quad$ Results}

We have run model 2 on two real board networks: the boards that are 1 degree of separation away from Chase Manhattan Bank's (CMB) board, and the boards 2 degrees of separation away from CMB's board. We refer to them in the following as CMB Net 1 and CMB Net 2, respectively. CMB Net 1 consists of 35 boards, with average size 12, and average fraction of shared directors equals to 0.1 . The average connectivity degree is 5 . CMB Net 2 consists of 277 boards, with average size 12 , and average degree 9 , average fraction of shared directors 0.1 .

We varied parameters $\alpha_{1}$ and $\alpha_{2}$ in order to understand what can be the impact of lobbies and the impact of information on the decision making process over the board network.

Results of simulations on CMB Net 1 and CMB Net 2 are given in figure 5 and figure $\mathbf{7}$ respectively.

For CMB Net 1, increasing the influence $\alpha_{1}$ of common appointments in external boards from 0 to 1 while keeping $\alpha_{2}=0$ (fig. $\mathbf{5}$ left), leads to the increase of $P^{+}$from $0.63 \pm 0.01$ to $0.68 \pm 0.01$. On the other hand $P^{+}$increases dramatically with the influence $\alpha_{2}$ of the information about connected boards' decisions (fig. 5 right). For high values of $\alpha_{2}$ there is no more dependence on $\alpha_{1}$.

When $\alpha_{1}=0$ and $\alpha_{2}=0$, then model 2 is formally equivalent to model 1. We recall that we have run model 1 on a network with the same topology as CBM Net1, but with an homogeneous fraction $\gamma$ of shared directors. When $\gamma$ equals the average fraction of shared directors observed in CBM Net1, then model 1 yields $P^{+}=0.6$, close to $P^{+}=0.63$ found with model 2 . So the etherogeneity of the fraction of shared directors doesn't seem to play a significant role on the network as a whole.

Figure 6 shows the map of the probability $P^{+}$to make decision +1 over the topology of the network. Dark nodes make decision +1 with high probability. Dark edges represent a board interlock consisting of two or more shared directors, clear edges represent one shared director interlock.

For CMB Net 2, there is no dependence of $P^{+}$on $\alpha_{1}$ even for $\alpha_{2}$ small. The dependence of $P^{+}$on $\alpha_{2}$ (fig. 7 , circles) is as dramatic as for CMB Net 1.

Of course the chosen schedule of meetings when information propagates from the center to the periphery seems important. To test this, we considered three different cases:

- CMB's board meets first, then the boards 1 degree of separation away from CMB's board meet with a fixed schedule and finally the boards 2 degrees away meet with a fixed schedule. 
- $\mathrm{CMB}$ meets first and then the other boards meet with a schedule randomly chosen at every run,

- the schedule of all boards, including the first, is randomly chosen at every run

Results obtained in the three cases are shown in figure 7 (circles, triangles and stars, respectively). The dramatic effect of information observed for case 1 (circles) is insensitive to the particular schedule chosen, inside layer 1 and inside layer 2 . In case 2 (triangles) the probability of making decision +1 remains small even when the influence of information is strong. In case 3 (stars) the probability of making decision +1 is basically not different from the control value 0.5 occurring when adoption of decision +1 and -1 are equally probable.

\section{Discussion and Conclusions}

We have presented two models for the decision making process of a network of interlocked boards about a common topic with a binary choice \pm 1 . The opinions of directors of the boards are initially evenly distributed, but the most central board meets first and takes the decision +1 by convention.

- Model 1 only assumes that agents are imitative and that directors with multiple appointments arrive at a board meeting with an initial opinion which favors the decision made in the last board meeting they participated at.

- Model 2 further takes into account the enhanced mutual influence of directors sharing appointments in outside boards and the decisions made by interlocked boards.

Results of simulations with model 1 show that the average probability $P^{+}$ that a board adopts the decision of the center node increases linearly with the average fraction $\gamma$ of directors shared by any two boards until it saturates. But the value of $\gamma$ observed in real board networks $(\gamma=0.1)$ corresponds to only 60 per cent chances to make decision +1 , compared to 50 per cent chances in absence of interlock. Therefore if one assumes that board interlock only introduces a bias in the initial opinions of interlocking directors, then the effect of the interlock on the decision making dynamics is very small.

For Model 2, we find that common appointments in external boards $\left(\alpha_{1}>0\right)$ affects very weakly the spreading dynamics. By contrast, information about interlocked boards' decisions $\left(\alpha_{2}>0\right)$ has a dramatic impact on the average probability $P^{+}$that a board adopts the decision of the center. If we assume that external information has an influence comparable to the influence of colleagues' average opinion $(\alpha 2 \simeq 0.1)$, we then find that almost certainly all boards end up adopting the decision of the center $\left(P_{+}=.9\right.$ for CMB Net $1, P_{+}=.9$ for CMB Net 2).

The order of boards meeting is of crucial importance: a meeting schedule respecting the distance of the boards from the central board is a necessary condition to have a large majority of boards making the same decision. In fact, 
innovations in real board networks has been observed [4] to start from a peripheral node and almost never from the most central node. But the diffusion takes place only when the central node adopts the innovation and it is then imitated by connected boards. In our model the radiation from the center hypothesis is based on the idea that the topic is discussed in a board when it is proposed by an interlocked director who has been involved in the same discussion on another board. Even if in real boards the meetings are not scheduled as we have assumed in our model, one can argue that directors of central boards usually know before the meeting what their board is likely to decide and through interlock this information can be taken into account in the meetings of more peripheral boards even if they are scheduled before the meeting of the central boards.

The models presented here are very stylized with respect to the real mechanisms involved in a decision making process in a network of boards. Our hypothesis about agents fall in the framework of the "herd behavior". We have chosen to make the smallest number of hypothesis on the agents in order to keep the behavior of the system completely comprehensible.

As a general conclusion we find that imitation of colleagues and opinion bias due to the interlock are not sufficient to trigger an avalanche of identical decisions over the network, whereas information about interlocked boards' decisions is. But there is no need to invoke public information about what all boards have decided, nor any external dramatic events.

This model thus provides a simple endogenous mechanism to explain the fact that boards of the largest corporations of a country can, in the span of a

few months, take the same decisions about general topics such as investments and advertisements.

\section{Acknowledgments}

We thank Gerald Davis (Univ. of Michigan), for having kindly provided the data of the US Fortune 1000 and for helpful comments. We also thank Jacques Lesourne and Andrea Bonaccorsi for fruitful discussion.

This work is supported by FET-IST department of the European Community, Grant IST-2001-33555 COSIN.

\section{References}

\section{References}

[1] Battiston, S., Bonabeau, E., Weisbuch G., Decision making dynamics in corporate boards, Physica A, 322, 567 (2003).

[2] Carpenter, M.A., Westphal, J.D., The strategic context of social network ties: examining the impact of director appointment on board involvement in strategic decision making, forthcoming in Academy of Management Journal. 
[3] Davis, G.F., Yoo, M., Baker, W.E., The small world of the American corporate elite, 1982-2001, Strategic Organization 1: 301-326 (2003).

[4] Davis, G.F. and Greve, H.R., Corporate elite networks and governance changes in the 1980s, Am. J. of Sociology, 103, 1-37 (1996).

[5] Fich E.M., White, L.J., Why do CEO's reciprocally sit on each other's boards?, forthcoming.

[6] Föllmer H., Random Economies with Many Interacting Agents, Journal of Mathematical Economics 1/1, 51-62 (1974).

[7] Galam S., Gefen Y. and Shapir Y., Sociophysics: A mean behavior model for the process of strike, Math. J. of Sociology, 9, 1-13 (1982).

[8] Galam S. and Moscovici S., Towards a theory of collective phenomena: consensus and attitude changes in groups, Eur. J. of Social Psychology, 21, 49-74 (1991).

[9] Haunschild, P.R., Interorganizational imitation: the impact of interlocks on corporate acquisition activity, Administrative Science Quarterly 38, 564-592 (1993).

[10] Orléan A., Bayesian interactions and collective dynamics of opinions: herd behavior and mimetic contagion, J. of Economic Behavior and Organization 28, 257-274 (1995).

[11] Weisbuch G. and Boudjema G., Dynamical aspects in the adoption of agri-environmental measures, Advances in Complex Systems, 2, pp. 11-36 (1999).

\section{$9 \quad$ Figures}




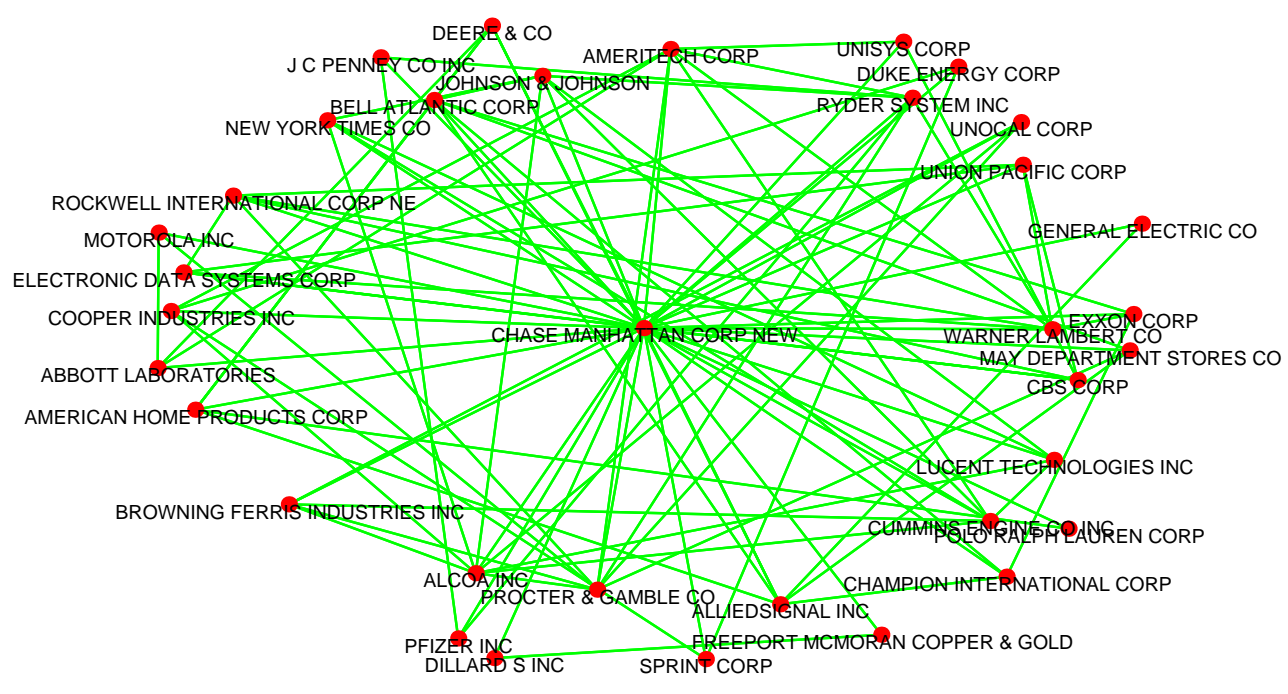

Figure 1: The network of boards at 1 degree of separation from Chase Manhattan Bank's board (CMB net 1).

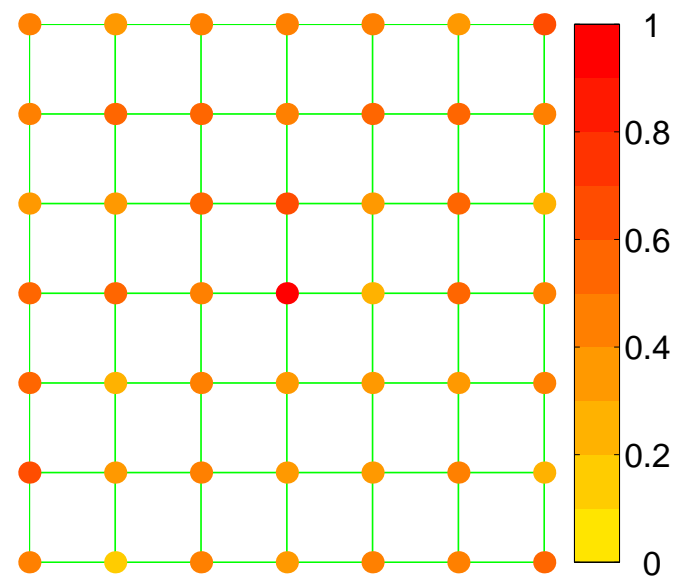

Figure 2: Square lattice of $7 \mathrm{X} 7$ nodes. A node represents a board of $n=10$ directors, a link represents the existence of some shared directors between two boards. Nodes color corresponds to the value of the probability $P^{+}$of adopting decision +1 , when the fraction $\gamma$ of shared directors between two connected boards is 0.4 . 


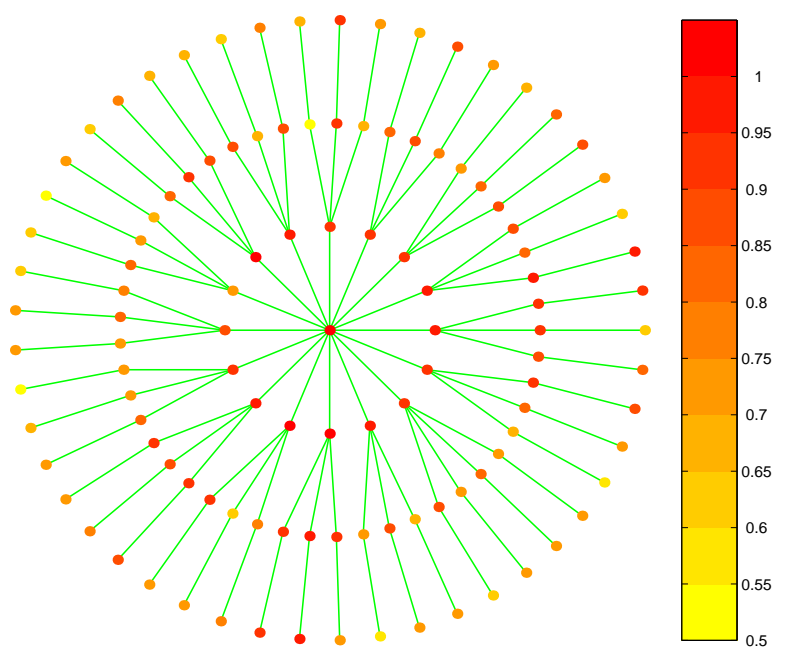

Figure 3: Star network of 115 nodes. The central node has connectivity degree 16 . The nodes in layers $2,3,4$ have connectivity degree 4,2,1 respectively. Nodes color corresponds to the value of the probability $P^{+}$of adopting decision +1 , when the fraction $\gamma$ of shared directors between two connected boards is 0.4 .

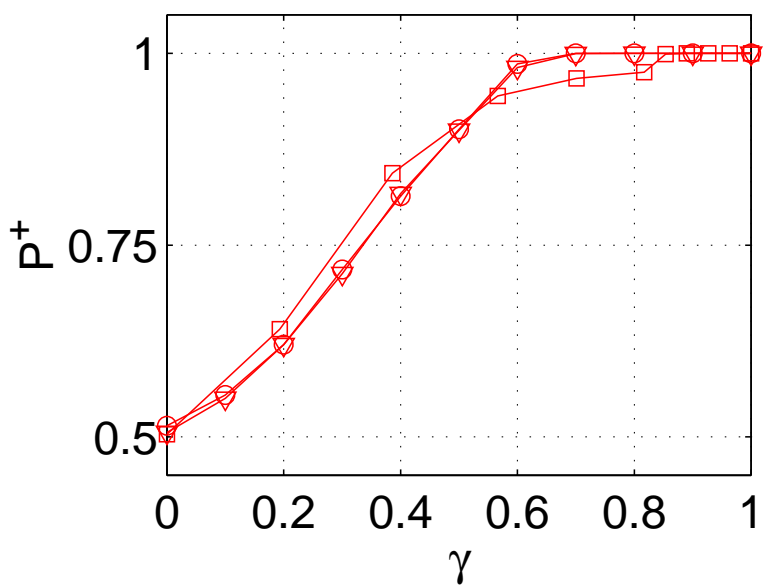

Figure 4: Probability of adoption of decision +1 as a function of the fraction $\gamma$ of shared directors between two connected boards. circles: lattice; triangles: star network; squares: the network CMB net 1 shown in figure 1 . 

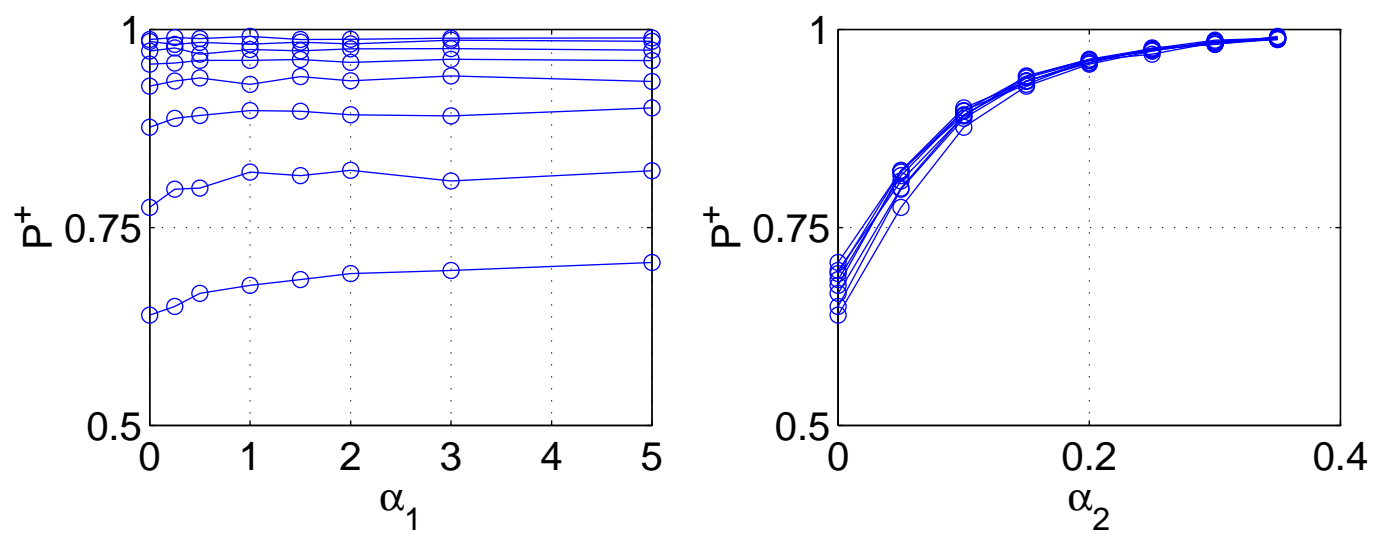

Figure 5: left: Probability $P^{+}$of making decision +1 as a function of the influence $\alpha_{1}$ of common appointments in outside boards. Each curve corresponds to a fixed value of the influence $\alpha_{2}$ of the information about connected boards' decisions. right: $P^{+}$as a function of $\alpha_{2}$.

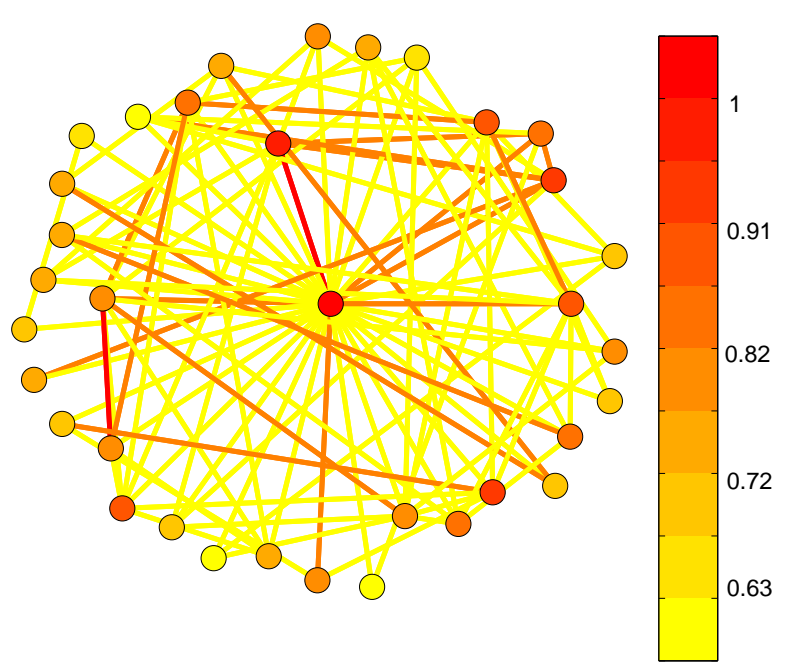

Figure 6: $\mathrm{CMB}$ net 1 . Color represents the probability $P^{+}$to make decision $+1 . P^{+}$ranges in $\left[\begin{array}{ll}0 & 1\end{array}\right] . P^{+}$is computed from 100 runs. Dark edges represent a board interlock consisting of two or more shared directors. Clear edges represent one shared director interlock. 


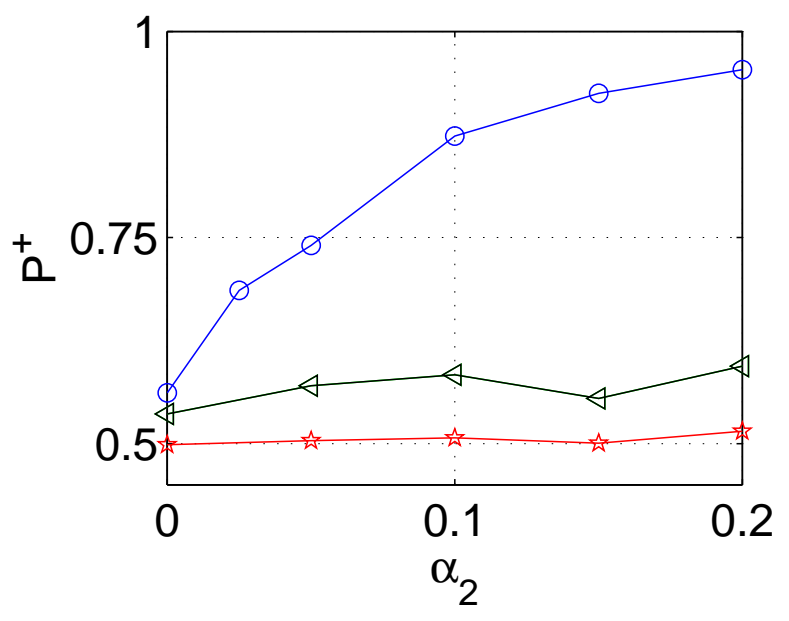

Figure 7: Probability $P^{+}$of adopting decision +1 as a function of the influence $\alpha_{2}$ of information about connected boards' decisions. Results on 100 runs. Three meeting schedules. Circles: first CMB, then boards at 1 degree of separation from CMB, then those at 2 degree of separation . Triangles: CMB meets first, then the other boards meet with a schedule randomly chosen at every run. Stars: the schedule of all boards, including the first, is randomly chosen at every run. 